\title{
Stimulus-determined and perceiver-determined aspects of haptic perceptual information processing
}

\author{
PAUL J. LOCHER \\ Montclair State College, Upper Montclair, New Jersey
}

\begin{abstract}
Speed of classification of stimuli haptically perceived without vision was measured in three experiments in which subjects sorted a deck of cards containing raised line stimuli generated from the orthogonal combination of two dichotomous attributes of lines: number and orientation. To enable assessment of the nature of the dimensional interaction for the set of stimuli, subjects in Experiment 1 performed 13 different speeded classification tasks of three basic types. Performance outcomes in these discrimination, focusing, and classification tasks indicated that differences in processing were due to the configural properties of the individual stimuli rather than to the dimensions used to generate the set of stimuli. Experiments 2 and 3 provided evidence that with extended practice and use of a holistic inspection strategy, subjects were able to modify the way they encoded and processed the stimuli in the focusing and condensation tasks. The role of stimulus-determined and perceiver-determined aspects of perceptual information processing as codeterminants of haptic perceptual performance is discussed.
\end{abstract}

Researchers have described ways in which dimensional interactions influence perceptual information processing. Reviews of this research have been presented by Garner $(1974,1983)$, who has made substantial contributions to the understanding of the stimulus-determined aspects of information processing. This area of research, however, has been limited to investigations of visual, and less frequently of auditory, stimuli and processes. I know of no research in which the nature of dimensional interactions defined in terms of converging experimental outcomes on a variety of perceptual tasks has been studied using stimuli haptically perceived without vision. In Experiment 1 of the present paper, I made an investigation of this type. In Experiments 2 and 3, I examined the extent to which task performance differences attributed to stimulus properties in Experiment 1 persisted when subjects were given attentional instructions and extended experience with the stimuli. The general purpose of the studies reported here, then, was to investigate both stimulus-determined and perceiver-determined aspects of haptic perceptual information processing.

\section{EXPERIMENT 1}

To assess the nature of the dimensional interaction for a set of stimuli, the influence of intrastimulus properties on processing must be distinguished from that of interstimulus properties. Garner (1978) demonstrated that this can be accomplished using 13 speeded classification tasks of three major types, described below. In Experiment 1, speed of classification of stimuli haptically perceived without vision was measured in these 13 tasks, and per-

Requests for reprints should be sent to P. Locher, Department of Psychology, Montclair State College, Upper Montclair, NJ 07043. formance outcomes were used to assess the nature of the dimensional interaction for the set of stimuli studied.

\section{Method}

Subjects. Ten right-hand-dominant undergraduates volunteered as subjects.

Stimuli. The four stimuli were generated from the orthogonal combination of two dichotomous attributes of lines: number and orientation. Stimuli consisted of either single or double lines that were either horizontal or vertical. Each stimulus was vacuum formed onto the center of a $3 \times 5$ in. $(7.6 \times 12.7 \mathrm{~cm})$ piece of Brailon which was backed with a filing card for ease of handling and sorting. The lines of the stimuli measured $10 \mathrm{~mm}$ in length and were raised $0.7 \mathrm{~mm}$. For double-line stimuli, the distance between the centers of the two lines was $5 \mathrm{~mm}$. The many investigations of the discriminability of tactual point symbols used to prepare tactual maps and displays for the blind (e.g., Schiff, 1980) were the bases for the selection of the stimulus attributes and their levels and the physical dimensions of the stimuli.

Tasks. In each of the 13 tasks used in this experiment, subjects sorted a deck of 36 cards into two piles as quickly and accurately as possible using haptic perception without vision. The tasks are described by denoting the four stimuli,,$- \mid,=, \|$, as A, B, C, D, respectively, and by indicating with a slash the appropriate stimulus groupings. Thus, Task A/B contained 18 cards each of A and B stimuli to be sorted into two separate piles. The 13 tasks were of three major types:

Discrimination tasks. The deck for these tasks contained just two stimuli, and subjects were required to discriminate between them. Two subtypes of discrimination tasks were formed by the six possible pairings of the four stimuli. Four single-attribute tasks involved discrimination between stimuli that differed on a single dimension: $A / B$ and $C / D$ (variation on orientation) and $A / C$ and $B / D$ (variation on number). Sorting times in these four tasks provided baseline data for all other tasks. The two correlated-attribute tasks, $\mathrm{A} / \mathrm{D}$ and $\mathrm{B} / \mathrm{C}$, required subjects to discriminate between stimuli that differed on both dimensions in a correlated or redundant manner.

Focusing tasks. The four focusing tasks, A/BCD, B/ACD, C/ABD, and $\mathrm{D} / \mathrm{ABC}$, required the classification of one stimulus versus the other three. In each task, the $\mathbf{1 8}$ focused stimuli in the deck were sorted into one pile and 6 each of the other stimuli were sorted into a second pile. Performance outcomes in these tasks identified those stimuli which, because of their particular configurations, were more or less easily processed than other stimuli in the set. 
Classification tasks. Classification tasks involved both dimensions. The deck, containing nine each of the four stimuli, was sorted into two classes of two stimuli each. Two types of classification tasks resulted from the ways in which the perceiver could pair the four stimuli to each of two responses. Filtering tasks were used to determine whether subjects selectively attend to one attribute while ignoring or filtering out the other. For Task AC/BD, subjects classified stimuli on the basis of orientation and filtered out the attribute number. For Task $A B / C D$, subjects attended to the attribute number and ignored orientation. For correct classification to occur in condensation task $\mathrm{AD} / \mathrm{BC}$, the perceiver had to process information from both attributes. Two stimuli forming a redundant pair were placed in each of two classes.

Procedure. Each subject was shown the four stimuli, and the attributes of the set were described. An explanation was given of each of the three major types of classification task and the sorting procedure used for each task; however, no strategy for exploring the raised line stimuli was demonstrated by the experimenter. For each data trial, the experimenter placed the deck of 36 cards into a tray which kept the cards face up in a pile. The tray rested on a small table and was covered by a box-like stand open in front and back. The subject placed his or her hands under the stand and, with the command to begin, sorted the deck into two piles based on the classification task-appropriate properties of the stimuli.

Subjects practiced each type of task until ready to begin the first data trial. The same basic exploratory strategy adopted by all subjects during practice was used throughout the experiment. With the left hand, the subject slid one card from the tray to the table under the stand. The stimulus was then scanned with the forefinger of the right hand with a broad back and forth and up and down scrubbing motion and then tossed into the appropriate pile, target stimuli to the right of the deck and nontarget stimuli to the left.

The experimental session, which included frequent rest periods, lasted approximately $2 \mathrm{~h}$, during which each subject performed a pseudorandom order of the 13 classification tasks three times. The orders of the tasks, counterbalanced across subjects, were constrained so that each type of task was distributed equally often throughout each set of tasks. After each trial, the experimenter recorded sorting time in 10ths of a second and gave this information to the subject. Typical of this type of task, errors were few and not recorded.

\section{Results}

The first block of trials was considered practice, and these data were not included in the analysis. Because a preliminary analysis indicated no significant interaction between blocks of trials and tasks, differences in task difficulty were examined by a within-subjects ANOVA performed on sorting times averaged over the second and third blocks of trials. It was found that the 13 task means in Table 1 are significantly different $[F(12,108)=6.68$, $p<.0001$, with a difference of $3.43 \mathrm{sec}$ between means being significant at $p<.01$ (Tukey HSD)].

Based upon the overall pattern of results presented in Table 1, differences in processing appear to be due to configural properties of the individual stimuli rather than to the dimensions used to generate the set of stimuli. This is particularly evident from the average sorting times for the focusing tasks. The D/ABC task was performed significantly more slowly than the other three focusing tasks, which were not significantly different from each other. These findings suggest that processing was carried out with properties of individual stimuli rather than dimensions.

Sorting times for the other tasks also indicate that the dimensions used to generate the set of stimuli were irrelevant in the processing of these stimuli and that subjects used the processing mode of focusing in all tasks. Although mean sorting times for the six discrimination
Table 1

Mean Sorting Times for the Discrimination, Focusing, and Classification Tasks of Experiment 1

\begin{tabular}{|c|c|c|c|c|c|}
\hline \multicolumn{2}{|c|}{ Discrimination Tasks } & \multicolumn{2}{|c|}{ Focusing Tasks } & \multicolumn{2}{|c|}{ Classification Tasks } \\
\hline Task & Time & Task & Time & Task & Time \\
\hline A/B & 46.30 & A/BCD & 46.58 & $\mathrm{AB} / \mathrm{CD}$ & 46.35 \\
\hline$C / D$ & 48.63 & $\mathrm{~B} / \mathrm{ACD}$ & 45.75 & $\mathrm{AC} / \mathrm{BD}$ & 49.98 \\
\hline $\mathrm{A} / \mathrm{C}$ & 45.43 & C/ABD & 46.55 & $\mathrm{AD} / \mathrm{BC}$ & 59.05 \\
\hline $\mathrm{B} / \mathrm{D}$ & 46.20 & $\mathrm{D} / \mathrm{ABC}$ & 50.60 & & \\
\hline $\mathrm{C} / \mathrm{B}$ & 45.32 & & & & \\
\hline $\mathrm{A} / \mathrm{D}$ & 46.53 & & & & \\
\hline
\end{tabular}

Note-Entries are mean times (in seconds) required to sort a deck of 36 cards into two piles.

tasks did not differ significantly, speed of discrimination was slower for the task of each subtype in which Stimulus D was paired with another stimulus. Thus, the same stimulus which provided the poorest focus also provided slower discrimination when paired with each of the other stimuli. Subjects had a particularly difficult time focusing Stimulus D when the basis of discrimination was the single-dimension orientation (Task C/D). Similarly, when asked to attend to the dimension orientation and ignore number in filtering Task AC/BD, performance was significantly slower than that in filtering Task $A B / C D$. Finally, the finding that the condensation task was performed significantly more slowly than all other tasks suggests that subjects focused on the configural properties of the individual stimuli and that processing was not based on the dimensions of the set of stimuli or on some emergent property resulting from perceptually fused dimensions.

\section{Discussion}

The importance of the individual stimulus in visual information processing tasks has been demonstrated by Garner (1978). He found that the stimulus (-) provided the easiest focus for a set of stimuli which also included (-(, )-(, and $)-)$, and that it also provided the easiest discrimination when placed with each of these three stimuli. According to Garner, the configural property of the "closed" stimulus (-) which facilitated processing is the gestalt concept of goodness. In his view, it is this property which makes a stimulus a good focus.

Why was Stimulus D a poor focus in the present study? Unlike the attributes of the stimuli used by Garner (1978), which are properties of stimulus parts (the individual parentheses) rather than properties of the stimulus as a whole, attributes of the stimuli used in the present research are properties of the whole stimulus. Orientation of a straight line is not confined to specific areas or parts of the line; it is a property of the whole stimulus. Since Stimuli C and D are composed of the same parts, focusing task differences in sorting speeds between these two stimuli cannot be due to differences in configuration of stimulus parts. Furthermore, no ready explanation of the data is available in terms of differences in levels of processing or the logical structure of the stimulus attributes investigated. If, for example, the levels of semantic processing for the two dimensions are assumed to differ, with orientation a more arbitrarily defined stimulus property than the more physical dimension, number, then one would expect an asymmetrical interaction of the dimensions (see Garner, 1983). The pattern of results reported in Table 1 does not, however, reflect process asymmetry.

Research has shown that the exploratory strategies used by blind and sighted individuals to direct haptic activity influence performance on haptic tasks (see, e.g., Schiff \& Foulke, 1982). This research has demonstrated that when subjects are initially exposed to braille, small complex dot patterns, or tactual point symbols, they typically use unsystematic movements of the fingers to explore the stimuli. With practice and training, exploratory strategies can be substantially improved, resulting in improved task performance. Simmons and Locher (1979) have shown that extended practice ( 384 test trials) interacting with the type of scan- 
ning strategy used to haptically explore without vision large nonrepresentational shapes increased subjects' abilities to detect symmetry.

The slower sorting speed in tasks involving Stimulus D in the present research may be an artifact of the exploratory strategy used to encode this stimulus. This seems to be the most reasonable explanation of the data, despite the fact that subjects were observed using the same scrubbing motion to explore all stimuli, and that Stimuli B and D were presented in the same orientation. Nevertheless, it is possible that the exploratory strategy used did not expose the finger to Stimulus D in the particular directions necessary for the most efficient stimulation of neural feature detectors (see, e.g., Sakata \& Iwamura, 1978). If Stimulus $\mathrm{D}$ provided a poor focus because it was inadequately represented in the organism due to an inefficient exploratory strategy, then with sufficient exposure to this stimulus, subjects may learn to focus more efficiently on the features of Stimulus D. This hypothesis was tested in the second study.

\section{EXPERIMENT 2}

\section{Method}

Ten right-hand-dominant college students, none of whom had participated in Experiment 1, volunteered as subjects. The stimuli, apparatus, card-sorting task, and procedures described in Experiment 1 were used in this study. The experimental session lasted approximately $1 \mathrm{~h}$, during which each subject performed a different random order of the four focusing tasks five times. Each subject, therefore, completed 720 test trials (36 stimuli sorted 20 times).

\section{Results}

The average sorting times in seconds for the four focusing tasks are shown in Figure 1 as a function of task trials. A 4 (task) $\times 5$ (task trial) repeated measures ANOVA performed on these data revealed significant main effects of task $[F(3,171)=61.88, p<.01]$ and trial $[F(4,171)=$ 109.27, $p<.001]$. The interaction was also significant $[F(12,171)=4.61, p<.01]$, and further analyses were performed on the data to specify the nature of the interaction.

For the first three sets of trials, subjects completed Tasks $A / B C D$ and $B / A C D$ significantly more quickly than Task C/ABD which was completed significantly more quickly than $\mathrm{D} / \mathrm{ABC}$ (all significant follow-up analyses are reported at $p<.01$ ). As in Experiment 1, Stimulus D provided the poorest focus in the first three sets of trials. The most important finding of Experiment 2 is that by the fifth set of trials, differences in sorting speeds between the four tasks were no longer significant. Thus, 576 focusing

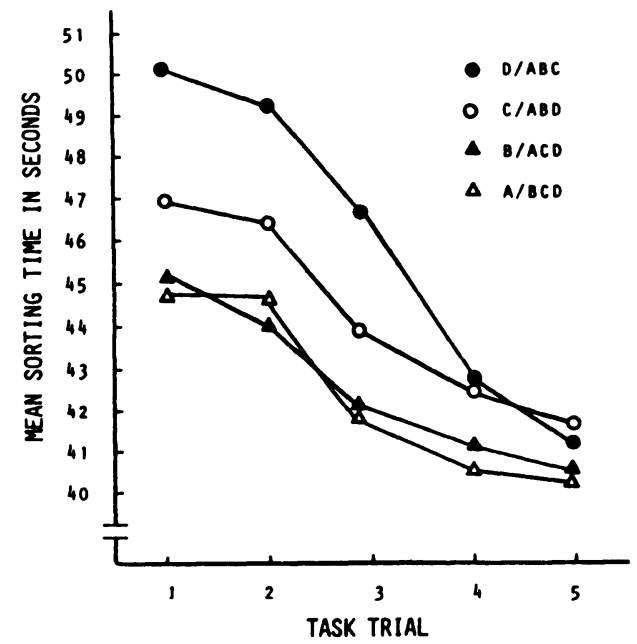

Figure 1. Mean sorting time in seconds across task trials for the four focusing tasks. task test trials provided subjects with sufficient experience with the stimuli so that all stimuli in the set became equally effective in allowing the use of a focusing strategy.

A significant increase in sorting speed in Task D/ABC had occurred by Trial 3, with an additional significant increase recorded for Trial 4. The increase in sorting times in the other three tasks, however, was not significant until Trial 4. Three different conclusions concerning subjects' abilities to selectively attend to the stimuli arise from the differential effects of experience across trials. The data for Trials 1,2 , and 3 indicate differences in selective attention due to the configural properties of individual stimuli. On Trial 4, Stimuli A and B were sorted more quickly than Stimuli $C$ and D; the difference in sorting times between Stimuli C and D was no longer significant, however. This result suggests an asymmetry of selective attention due to stimulus dimensions. Finally, no significant differences in subjects' abilities to focus on each of the stimuli was observed on Trial 5. When one considers the different interpretations of the data in Experiment 1, which would result from the inclusion of each of the three focusing task outcomes observed in Experiment 2, the need to systematically investigate the effects of experience in research concerned with stimulus-determined aspects of perceptual processing becomes apparent.

\section{Discussion}

Garner (1978) reported a practice effect for the visual modality in 1 of a series of 11 experiments, each of which included the 13 speeded classification tasks used here in Experiment 1. Garner's subjects showed significant improvement in sorting speed in the condensation task relative to the filtering tasks between the second and third blocks of trials. This finding, plus his observation that the literature at that time contained little evidence of the effect of learning on how stimulus elements are perceived, prompted Garner to point out the need to investigate fully the extent to which learning influences perceptual information processing. To date, however, this issue has been almost completely neglected by researchers. In fact, the majority of investigations of the nature of perceptual processing of multidimensional stimuli do not provide subjects wih sufficient practice with a set of stimuli to permit the effects of learning upon processing to become manifest. The results of Experiment 2 clearly indicate that the effects of learning must become an important and frequent experimental variable in such studies.

\section{EXPERIMENT 3}

Experiment 3 was conducted to explore further the effect of stimulus familiarity on haptic processing of stimulus attributes. In addition to the variable experience, Experiment 3 also examined the influence of type of exploratory strategy used to encode the stimulus upon the speed of classification in a condensation task.

In Experiment 1, condensation task performance was significantly slower than that in any of the other 12 tasks. The relative difficulty of performance in the condensation task as contrasted to the filtering tasks suggests that classification was carried out on the basis of perceptually distinct properties of the stimuli rather than by selective attention to an emergent configural property resulting from perceptually fused dimensions. One explanation for subjects' poor condensation task performance in Experiment 1 is that they were unable to perceive an emergent property because the scrubbing motions used to explore 
the stimuli did not permit them to encode the stimuli holistically. Poor braille readers, for example, often engage in this type of scrubbing exploratory motion (Foulke, 1982). If this explanation is correct, then use of a holistic inspection strategy which permits subjects to attend to information from both dimensions as a unit should make the condensation task, with practice, less difficult than when the scrubbing strategy is employed. This hypothesis was tested in the present experiment.

The holistic exploratory strategy used by one half of the subjects in this study was suggested by the many investigations of the aspects of hand and finger movements which contribute to efficient reading of braille and tactual point symbols by the blind.

\section{Method}

Twenty right-hand-dominant college students, none of whom had participated in the previous experiments, volunteered as subjects. The stimuli, apparatus, card-sorting task, and procedures described in Experiment 1 were used during a 1-h experimental session.

Two groups of 10 subjects each sorted the deck 20 times while performing the condensation task. Prior to the first data trial, both groups of subjects were told that they would be given their sorting time after each trial, and their task was to try on each successive trial to sort the deck slightly faster without making errors. One group was not shown a strategy to be used to explore the stimuli. It was expected that subjects in this group would spontaneously use the same scrubbing strategy as that employed by subjects in Experiments 1 and 2. Observation of the group's exploratory activity confirmed this expectation.

In addition to the information given the first group prior to the first data trial, the experimenter described and demonstrated the strategy which the second group of subjects was to use to explore the stimuli. This holistic strategy was performed by pressing the forefinger of the right hand over the raised lines and simultaneously rolling the finger tip slowly and with constant motion over the stimulus. To avoid creating an attention set, the term holistic strategy was not used with subjects.

\section{Results and Discussion}

To determine the influence of practice and exploratory strategy on sorting time, the data were entered into a 2 (type of strategy) $\times 5$ (blocks of trials) mixed ANOVA. For this analysis, data for the $\mathbf{2 0}$ trials were collapsed into five blocks. The dependent variable, therefore, was average sorting time per block of four trials per subject. The average sorting times in seconds for Blocks 1 through 5 were $81.27,66.84,60.70,56.75$, and 56.12 , respectively, for subjects who used the scrubbing strategy, and 78.44, $62.28,52.34,49.00$, and 44.48 , respectively, for those who used the holistic strategy.

No significant main effect of type of strategy was found $[F(1,18)=2.41]$. The main effect of blocks of trials $[F(4,72)=84.16, p<.001]$ and the interaction $[F(4,72)$ $=6.41, p<.01]$ were significant. Follow-up tests revealed that condensation task performance significantly improved with practice for both groups of subjects. Use of the scrubbing strategy resulted in a significant reduction in average sorting speeds beween Blocks 1 and 2 $(p<.01)$ and Blocks 2 and $4(p<.05)$. Subjects who used the holistic strategy significantly reduced their sorting times between Blocks 1 and $2(p<.01)$, Blocks 2 and $3(p<.05)$, and Blocks 3 and $5(p<.05)$.

Performance between the two groups of subjects did not differ for the first two blocks of trials. However, by the third block, use of the holistic strategy resulted in an average sorting speed which was $8.36 \mathrm{sec}$ faster than that recorded for subjects who used the scrubbing strategy. This difference is significant at $p<.05$, as is the $7.75 \mathrm{sec}$ difference between the groups recorded for Block 4. By the fifth block of trials, the difference in sorting times between the groups, which was now $11.63 \mathrm{sec}$, was significant at the .01 level. Thus, while extended experience with the set of stimuli sigificantly decreased sorting speed for both groups of subjects, those who used the holistic encoding strategy were better able, with practice (approximately 300 test trials), to attend to both stimulus dimensions than were subjects who used the scrubbing strategy. As hypothesized, experience and type of strategy used to encode the stimuli functioned together to facilitate haptic perception of stimulus attributes in the condensation task.

Average sorting speed for the fifth block of condensa-

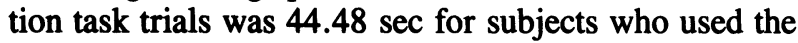
holistic strategy in this experiment. This value is very similar to average sorting times for the four focusing tasks on Trial 5 in Experiment 2 and for the six discrimination tasks in Experiment 1. This similarity suggests that the significant differences in sorting speeds between the 13 tasks observed in Experiment 1 might no longer exist if subjects were permitted sufficient exposure to the set of stimuli and instructed to use a holistic strategy to encode the stimuli. An outcome such as this would require a different conclusion concerning the nature of the stimulus dimensions of the set of stimuli studied than that reached in Experiment 1. Results of the three experiments demonstrate that the nature of the dimensional interaction for a set of stimuli haptically perceived cannot be adequately assessed unless one investigates both intrastimulus and interstimulus properties of the stimulus ensemble, as well as the effect of learning and other organismic or perceiver-determined aspects of processing.

\section{REFERENCES}

FoulKE, E. (1982). Reading braille. In W. Schiff \& E. Foulke (Eds.), Tactual perception: A sourcebook (pp. 168-208). Cambridge: Cambridge University Press.

GARNER, W. (1974). The processing of information and structure. Potomac, MD: Erlbaum.

GARNER, W. (1978). Selective attention to attributes and to stimuli. Journal of Experimental Psychology: General, 107, 287-308.

GARNER, W. (1983). Asymmetric interactions of stimulus dimensions in perceptual information processing. In T. Tighe \& B. Shepp (Eds.), Reception, cognition, and development: Interactional analyses (pp. 138). Hillsdale, NJ: Erlbaum.

SAKata, H., \& Iwamura, Y. (1978). Cortical processing of tactile information in the first somatosensory and parietal association areas in the monkey. In G. Gordon (Ed.), Active touch (pp. 55-72). New York: Pergamon Press.

SCHIFF, W. (1980). Perception: An applied approach. Boston: Houghton Mifflin.

SCHIFF, W., \& FoULKE, E. (1982). Tactual perception: A sourcebook. Cambridge: Cambridge University Press.

Simmons, R., \& LoCHER, P. (1979). Role of extended perceptual experience upon haptic perception of nonrepresentational shapes. Perceptual \& Motor Skills, 48, 987-991.

(Manuscript received for publication December 5, 1985.) 